\title{
Epidemiological and cohort study finds no association between COVID-19 and Guillain-Barré syndrome
}

Stephen Keddie, ${ }^{1,2}$ Julia Pakpoor, ${ }^{3}$ Christina Mousele, ${ }^{2}$ Menelaos Pipis, ${ }^{1,2}$ Pedro M. Machado, ${ }^{1,2}$ Mark Foster, ${ }^{2}$ Christopher J. Record, ${ }^{4}$ Ryan Y. S. Keh, ${ }^{5}$ Janev Fehmi, ${ }^{6}$ Ross W. Paterson, ${ }^{2,7}$ Viraj Bharambe, ${ }^{8}$ Lisa M. Clayton, ${ }^{9}$ Claire Allen, ${ }^{10}$ Olivia Price, ${ }^{11}$ Jasmine Wall,,${ }^{5}$ Annamaria Kiss-Csenki, ${ }^{12}$ Dipa P. Rathnasabapathi, ${ }^{13}$ Ruth Geraldes, ${ }^{6,14}$ Tatyana Yermakova, ${ }^{15}$ Joshua King-Robson, ${ }^{16}$ Maya Zosmer, ${ }^{17}$ Sanjeev Rajakulendran, ${ }^{2,17}$ Sheetal Sumaria, ${ }^{2}$ Simon F. Farmer, ${ }^{2}$ Ross Nortley, ${ }^{2,14}$ Charles R. Marshall, ${ }^{9}$ Edward J. Newman, ${ }^{18}$ Niranjanan Nirmalananthan, ${ }^{4}$ Guru Kumar, ${ }^{7}$ Aswin A. Pinto, ${ }^{13}$ James Holt, ${ }^{8}$ Tim M. Lavin, ${ }^{19}$ Kathryn M. Brennan, ${ }^{18}$ Michael Zandi, ${ }^{2}$ Dipa L. Jayaseelan, ${ }^{2,20}$ Jane Pritchard, ${ }^{21}$ Robert D. M. Hadden, ${ }^{16}$ Hadi Manji, ${ }^{1,2}$ Hugh J. Willison, ${ }^{18}$ Simon Rinaldi, ${ }^{6}$ Aisling S. Carr ${ }^{2}$ and Michael P. $\operatorname{Lunn}^{1,2}$

Author affiliations:

1 Department of Neuromuscular Diseases, University College London, London, UK

2 National Hospital for Neurology and Neurosurgery, University College London Hospitals NHS Foundation Trust, London, UK

3 Oxford School of Public Health, Oxford, UK

4 St George's University Hospitals NHS Foundation Trust, London, UK

5 Lancashire Teaching Hospitals NHS Foundation Trust, UK

6 Nuffield Department of Clinical Neurosciences, University of Oxford, Oxford, UK

7 Darent Valley Hospital, Dartford, UK

8 The Walton Centre National Health Service (NHS) Foundation Trust, Liverpool, UK

9 Barts Health NHS Trust, London, UK

10 Poole Hospital NHS Foundation Trust, Poole, UK

11 Basildon and Thurrock University Hospital Trust, Basildon, UK

12 Hampshire Hospitals NHS Foundation Trust, Hampshire, UK 
13 Wessex Neurological Centre, University Hospital Southampton NHS Foundation Trust, Southampton, UK

14 Wexham Park Hospital, Frimley Health Foundation Trust, Berkshire, UK

15 Leeds Teaching Hospitals NHS Trust, Leeds, UK

16 Kings College Hospital NHS Foundation Trust, London, UK

17 North Middlesex University Hospital NHS Trust, London, UK

18 Queen Elizabeth University Hospital, Glasgow, UK

19 Manchester Centre for Clinical Neuroscience, Salford Royal Hospital NHS Foundation Trust, Manchester, UK

20 West Hertfordshire Hospitals NHS Trust, Watford, UK

21 Imperial College Healthcare NHS trust, London, UK

Correspondence to: Professor Michael P. Lunn

Centre for Neuromuscular Disease, National Hospital for Neurology and Neurosurgery, Queen Square, London, WC1N 3BG, UK

E-mail:michaellunn@nhs.net

Running title: No association between COVID-19 and GBS

\begin{abstract}
Reports of Guillain-Barré syndrome (GBS) have emerged during the Coronavirus disease 2019 (COVID-19) pandemic. This epidemiological and cohort study sought to investigate any causative association between COVID-19 infection and GBS. The epidemiology of GBS cases reported to the UK National Immunoglobulin Database was studied from 2016 to 2019 and compared to cases reported during the COVID-19 pandemic. Data were stratified by hospital trust and region, with numbers of reported cases per month. UK population data for COVID19 infection were collated from UK public health bodies. In parallel, but separately, members of the British Peripheral Nerve Society prospectively reported incident cases of GBS during
\end{abstract}


the pandemic at their hospitals to a central register. The clinical features, investigation findings and outcomes of COVID-19 (definite or probable) and non-COVID-19 associated GBS cases in his cohort were compared. The incidence of GBS treated in UK hospitals from 2016 to 2019 was $1.65-1.88$ per 100000 individuals per year. In 2020, GBS and COVID-19 incidences varied between regions and did not correlate with one another $(r=0.06,95 \%$ confidence interval: -0.56 to $0.63, P=0.86$ ). GBS incidence fell between March and May 2020 compared to the same months of 2016-19. In an independent cohort study, 47 GBS cases were reported (COVID-19 status: 13 definite, 12 probable, 22 non-COVID-19). There were no significant differences in the pattern of weakness, time to nadir, neurophysiology, CSF findings or outcome between these groups. Intubation was more frequent in the COVID-19 affected cohort (7/13, 54\% versus 5/22, 23\% in COVID-19-negative) likely related to COVID-19 pulmonary involvement. Although it is not possible to entirely rule out the possibility of a link this study finds no epidemiological or phenotypic clues of SARS-CoV-2 being causative of GBS. GBS incidence has fallen during the pandemic, which may be the influence of lockdown measures reducing transmission of GBS inducing pathogens such as Campylobacter jejuni and respiratory viruses.

Keywords: Guillain-Barré syndrome; SARS-CoV-2; COVID-19

Abbreviations: COVID-19 = Coronavirus disease 2019; GBS = Guillain-Barré syndrome; IGOS = International GBS Outcome Study; IVIg = intravenous immunoglobulin; NHSE = NHS England; SARS-CoV-2 = severe acute respiratory syndrome coronavirus 2

\section{Introduction}

The first cases of severe acute respiratory syndrome coronavirus 2 (SARS-CoV-2) were reported to the WHO in late 2019, and by March 2020 COVID-19 was pandemic. ${ }^{1}$ SARS-CoV and Middle East respiratory syndrome coronavirus (MERS-CoV) were associated with neurological sequelae. ${ }^{2}$ Early reports identified neurological symptoms of COVID-19 infection as fever, headache, anosmia and dysgeusia. ${ }^{3}$ Subsequently COVID-19 infection has been associated with stroke, meningoencephalitis, acute disseminated encephalomyelitis and Guillain-Barré syndrome (GBS). ${ }^{4-6}$ The first reported case of GBS questioned a possible link with COVID-19 and occurred in late January 2020 in a COVID-19 asymptomatic patient who 
developed COVID-19 symptoms at Day 8 of GBS. ${ }^{7}$ The first series of five patients with GBS following SARS-CoV-2 infection was reported in April 2020, ${ }^{8}$ followed by case reports, case series and collective reviews.

GBS is an acute, post-infectious immune mediated polyradiculoneuropathy typically arising a few days to 6 weeks after bacterial or viral infections including Campylobacter jejuni, Haemophilus influenzae, Mycoplasma pneumoniae, influenza, Epstein-Barr virus, cytomegalovirus, and more recently, Zika virus. ${ }^{9-11}$ The pathogenesis of GBS following the majority of presumed causative infectious is unknown, although humoral molecular mimicry is definitively established for $C$. jejuni-associated GBS, and may play a role in many or most cases of GBS. ${ }^{12,13}$ The pathological initiating event of $C$. jejuni GBS is the manufacture of antibodies to lipo-oligosaccharide surface epitopes of $C$. jejuni that cross-react with peripheral nerve glycolipids, resulting in complement fixation, macrophage attraction and resultant peripheral axon or myelin nerve damage. ${ }^{14}$ This mechanism may also occur with other bacterial and viral pathogens since anti-ganglioside antibodies are found in up to $60 \%$ of GBS cases, ${ }^{15}$ including those associated with viral infections; for example, anti-GM2 antibodies occur in CMV-associated GBS. GBS associated with ganglioside complexes increases the frequency of potential ganglioside related molecular mimicry, and the presence of antibodies to paranodal and juxtaparanodal antigens suggests an unproven post-infectious link to protein epitopes. ${ }^{16,17}$ Zika-associated GBS has such a close association to seroconversion that some cases may be due to direct but unproven neurotropic damage.

SARS CoV-2 is a single-stranded RNA enveloped virus. Open reading frames (ORF) encode for replicase proteins and the structural proteins which are the spike $(\mathrm{S})$, nucleocapsid $(\mathrm{N})$, envelope (E) and membrane (M) proteins. ${ }^{18}$ To date we know of no homology between SARSCoV-2 surface epitopes and peripheral nerve tissue. Reports of varied anti-ganglioside antibodies in association with COVID-19 GBS suggest that a uniform CMV-like immunemediated hypothesis is unsupported. More comprehensive epidemiological characterization is crucial to understanding any causal link. With over 33 million cases of COVID-19 infection worldwide by 29 September $2020,{ }^{19}$ the question of whether COVID-19 infection is a cause of GBS or a coincidental finding remains to be answered.

This study aimed to investigate whether a causal relationship could be determined between COVID-19 and GBS, and was performed in three parts. First, we retrospectively explored UK population-based epidemiological datasets of cases with confirmed COVID-19 and compared 
that to patients hospitalized with GBS. Separately, but in parallel, we characterized a large cohort of the incident UK GBS cases presenting both with, and without, COVID-19 to explore timing of onset, and any identifying phenotypic characteristics that might hint at a specific mechanistic link (as for example in sensory GBS associated with CMV). Finally, we explored any homology between SARS-CoV-2 and the human genome and proteome that would support a molecular mimicry mechanism.

\section{Materials and methods}

\section{Epidemiological case reporting}

Incident hospitalized cases of GBS were retrospectively ascertained from the UK National Immunoglobulin Database from the 1 January to 31 May 2020, demonstrating the frequency of GBS cases across the year, pre and during the COVID-19 pandemic. NHS England (NHSE) procures the total intravenous immunoglobulin (IVIg) supply for England, Scotland and Northern Ireland. NHSE mandates that every IVIg prescription is approved by a clinical panel and is reported onto the database within 90 days. Recording compliance is almost $100 \%$ as hospital trusts are only reimbursed once records of dispensed volumes are submitted; these are retrospectively cross-checked against supply and returned stocks. ${ }^{20}$ To ensure complete reporting of cases, NHSE specifically mandated all users of the National Immunoglobulin Database to log any outstanding GBS cases by 30 June 2020 by email on 9 June 2020. Data retrieval was then performed on 7 July 2020 to allow time for reporting delay.

Current UK guidance for GBS treatment indicates IVIg or plasma exchange (PLEX) as first line therapy, ${ }^{21}$ but IVIg is, in practice, first line in most UK hospitals as PLEX is normally not as available. IVIg is also only authorized in the UK for patients with Hughes Grade $\geq 4$, progressing towards intubation and ventilation, with a high likelihood of respiratory support (mEGRIS score $\geq 3$ ) or a predicted poor prognosis (mEGOS $\geq 4$ ). The patients usually treated with IVIg in the UK are those who require admission, and although this under-ascertains the true incidence of GBS, it reduces the effects of attendance bias in a pandemic. IVIg is given to nearly all presenting GBS patients in Europe as illustrated by $86 \%(612 / 715)$ of European cases treated with IVIg in the International GBS Outcome Study (IGOS), ${ }^{22}$ and 88\% (37/42) of 'COVID-19 GBS' in the literature until July. ${ }^{23}$ 
We also searched the NHSE Immunglobulin Database for GBS cases from 1 January to 31 May in each of the years 2016 to 2019) to determine the incidence of non-COVID-19 reported cases of GBS to compare to the 2020 pandemic data. Data were stratified by hospital trust and region. UK population data for COVID-19 PCR confirmed infection were collated from Public Health England, Health Protection Scotland and the Public Health Agency of Northern Ireland. Because of the lack of testing available testing early in the pandemic, COVID-19 PCR confirmed cases were significantly fewer than the true incidence of infection across the UK. ${ }^{24}$ Thus in addition, we obtained data from the NHS Blood Transfusion Service (NHSBT) ${ }^{25}$ of antibody seroprevalence across the UK to SARS-CoV-2, and used the London data to study the number of GBS cases that occurred compared both to the number of PCR confirmed cases and the number of seroconverted COVID-19 cases during the pandemic months.

\section{Cohort study}

In parallel to the epidemiological study, we conducted a prospective cohort study to compare the demographic, phenotypic and infective associations of COVID-19 associated GBS (definite and probable) to COVID-19 negative GBS reported during the same study period.

Reports of GBS were submitted by members of the British Peripheral Nerve Society (BPNS), who cover 81 different UK sites. Members were emailed on a weekly basis to collect information on hospital presentations of GBS from 1 March to 31 May 2020. Reporting was restricted to BPNS members to achieve a comprehensively characterized representative sample of incident cases diagnosed by peripheral nerve experts. Data were entered to the International Neuromuscular COVID-19 database (www.ucl.ac.uk/centre-for-neuromusculardiseases/news/2020/may/international-neuromuscular-covid-19-database), at the Centre for Neuromuscular Disease. Cohort study data collection ended on 1 July 2020 to allow time for retrospective case reporting. Anonymized clinical data of demographics and medical history, COVID-19 infection, symptoms and management were collected. Precipitating illness, clinical features of GBS, investigation findings including CSF and electrophysiology, management and outcomes were also collated.

Data collected from the cohort study were compared to the phenotypic characteristics of the published European International GBS Outcome Study cases to assess whether pandemic presentations differed from a comparable cohort of non-pandemic phenotypes. $^{22}$ 


\section{Evidence of COVID-19}

GBS cases were stratified into three groups: definite COVID-19, probable COVID-19 and nonCOVID-19. Definite cases had either a positive nasal or throat swab PCR for viral RNA or a subsequent positive serological test for anti-SARS-CoV-2 IgM or IgG irrespective of clinical signs and symptoms. Probable cases were defined by the presence of clinical symptoms consistent with COVID-19 infection as per the European Centre for Disease Prevention and Control case definitions, ${ }^{26}$ or pulmonary imaging [chest X-ray (CXR) or CT] highly suggestive of COVID-19 (airway opacification typically bilateral, peripheral and basal in distribution) where PCR analysis was negative. Occurrence of GBS within 6 weeks of acute COVID-19 infection (clinically or on confirmed laboratory findings) was considered necessary to confirm a definite link, with longer timeframes accepted but recorded in the data and classified as probable. $^{27}$

\section{Search for homology between SARS-CoV-2 and human genome and proteome}

At the time of this study relatively little is known of the epitope presentation and immunobiology of SARS-CoV-2. We searched for evidence of molecular mimicry between any SARS-CoV2 proteins and human nerve axonal or myelin proteins and glycoproteins, recognizing that epitopes are not all protein and not necessarily all linear. We searched for human homologues of proteins encoded by the SARS-CoV-2 genome using the National Centre for Biotechnology Information (NCBI's) Basic Local Alignment Search Tool (BLAST) to identify common amino acid sequences in the human Reference Sequence Database (refseq_protein). The NCBI BLAST was also used to query the SARS-CoV-2 genome against the human genome for any significant alignments at specific genomic loci.

The expect value (E-value) quantifies the number of times a specific alignment can be 'expected' to occur in a database by chance. As the E-value decreases the significance of the alignment in the specified database increases. Any alignment with an E-value of $\leq 1 \times 10^{-4}$ was considered homologous to a human protein (error rate $<0.01 \%$ ).

\section{Statistical analysis}

The incidence rates of GBS and COVID-19 (95\% confidence intervals by Byar's approximation method $)^{28}$ were calculated by dividing regional cases and time period by the 
relevant mid-year population estimate. Mid-year population estimates at both regional and national level were obtained from the UK Office for National Statistics. ${ }^{29}$ We explored any association between the incidence of GBS and the incidence of COVID-19 in UK regions in 2020 (January to May) using Pearson's correlation coefficient. The Shapiro-Wilk test was used to determine suitability of parametric tests.

COVID-19 definite and probable cases in the cohort were statistically compared against nonCOVID-19 associated GBS. In addition, to determine whether characteristics differed between pandemic and non-pandemic GBS phenotypes, clinical characteristics of our study cohort were also compared to published IGOS study participants. We used Mann-Whitney U to test nonparametric continuous data, and the $\chi^{2}$ or Fisher's exact test to compare proportions. IGOS data stratified to European/American cases $(n=715)$ were used in preference to the entire IGOS cohort $(n=925)$ where available. ${ }^{22} \mathrm{R}(4.0 .0)$ and GraphPad prism (8.1.2) were used for analysis and figures.

\section{Ethics}

The UK Health Research Authority was consulted and advised the study did not require review by an NHS Research Ethics Committee as an analysis of previously collected non-identifiable information. The project was submitted as a 'Service Evaluation' to the Clinical Audit and Quality Improvement Subcommittee (CAQISC).

\section{Data availability}

Data are available upon the request to the corresponding author.

\section{Results}

\section{Epidemiological study}

The NHSE Immunoglobulin Database reported a mean of 1098 (range 1021-1155) GBS cases per year in the UK (excluding Scotland) between 2016 and 2019 (monthly range 83-170 cases). This represents the UK GBS population who are admitted to hospital with GBS and can be treated. As PLEX is seldom used and only patients with significant GBS are treated, IVIg treatments represent the vast majority of UK cases. Annual UK GBS incidence requiring 
treatment was therefore a minimum of $1.65-1.88$ per 100000 individuals each year across this period, consistent with the incidence of GBS in Europe and North America from a previous meta-analysis of 1643 GBS cases (range $0.81-1.89$ cases per 100000 ). ${ }^{30}$ These comparative figures along with the mandatory reporting supports the NHSE Immunoglobulin Database as the most comprehensive, complete and accurate resource for epidemiological analysis of GBS in the UK.

Although COVID-19 was first reported in the UK on 31 January 2020, significant numbers of daily new infections in the first wave ( $>1000$ per day) did not occur until March, with the highest recorded daily count of 6201 confirmed cases prior to this report on 1 May $2020 .{ }^{31}$ Through April and May there were between 4000 and 6000 COVID-19 cases per day. If a strong causative and temporal association existed, COVID-19 GBS cases would be expected to rise in subsequent weeks (Fig. 1). However, even accounting for the consistent summer dip in GBS cases seen in 2016-2019, GBS cases in March (93), April (70) and May (56) of 2020 were significantly fewer than years 2016-19 [mean 132 (March), 116 (April) and 113 (May)] (Fig. 2). GBS and COVID-19 incidences varied across UK regions with no correlation between COVID-19 and GBS at a regional level ( $r=0.06095 \%$ confidence interval -0.56 to $0.63, P=$ 0.86) (Fig. 3 and Supplementary Table 1).

By the 1 March 2020 there were only 17 PCR-positive confirmed COVID-19 cases in London, which increased to 26798 by the 27 April $2020 .{ }^{31}$ In London there were 25 cases of GBS registered to the NHSE IVIg database during this time (138 in the rest of the UK). Using these figures for this period, the estimated occurrence rate is 0.82 GBS cases per 1000 COVID-19 infections. However, serological data from London blood donors on the 27 April 2020 reported the prevalence of prior SARS-CoV-2 infection in London as $17.5 \%,{ }^{25}$ equivalent to 1571850 individuals having made a serological response to COVID-19. This is more likely to give the true estimate of COVID-19 infections in the community. Using 1571850 as the denominator for infection, the occurrence rate of GBS is more likely to be 0.016 cases per 1000 COVID-19 infections.

\section{Cohort study}

Forty-seven cases of GBS were reported to the cohort by BPNS members over a 12-week collection period. Patients were classified according to the Brighton Criteria ranging from high to low of diagnostic certainty. ${ }^{32}$ Twenty-two were level 1 (46\%) and 15/47 (32\%) level 2 with 
four level 3 and six (13\%) level 4, similar to previously reported large GBS cohorts. ${ }^{33}$ Of the 47 cases, 13 had definite COVID-19 infection, 12 were probable, and 22 had GBS with no evidence of COVID-19. Median age was 57 years [interquartile range (IQR 19-88)], 33 were male (70\%) and 29 (66\%) were Caucasian. The male:female ratio in the COVID-19 patients was 5.5 compared to 1.4 in the non-COVID and IGOS study. Males are more likely to be significantly unwell and hospitalized with COVID-19 infection, which may partially or completely explain this difference. ${ }^{34,35}$

The clinical characteristics of the patients with GBS are shown in Table 1 and Supplementary Table 2. For patients with COVID-19 infection, the median time between onset of infective symptoms and neurological weakness was 12 days for definite COVID-19 and 5 days for probable cases; however, the range of time intervals was very broad, ranging from 0 to 37 days in definite and -14 to 52 days in probable cases, with only one case in which GBS developed over 6 weeks following COVID-19 onset. Three probable cases developed symptoms of GBS without any clear COVID-19 symptoms, and had incidental imaging evidence of COVID-19 suggesting recent mild or asymptomatic infection. Numbers of non-COVID-19 GBS cases with symptoms of a precipitating illnesses, particularly gastroenteritis, were significantly fewer than that compared to the IGOS cohort [1/22, 5\% in non-COVID-19 cases compared to $163 / 652$ $(25 \%)$ in the IGOS cohort, $P<0.000]$. This may be an effect of lockdown with improved hand hygiene reducing numbers of faecal-oral pathogen transmissions. The pattern of weakness and time to nadir were no different between COVID-19 associated GBS and non-COVID-19 GBS. Cranial nerve involvement was the only finding more frequent in the IGOS study compared to our cohort. Although not statistically significant, electrophysiological studies found a higher proportion of axonal GBS in the non-COVID-19 patients [four acute motor and sensory axonal neuropathy (AMSAN) and one acute motor axonal neuropathy (AMAN) 23\%, compared to one with AMSAN only from the COVID-19 positive group].

The use of ventilation did not differ significantly between COVID-19 (definite and probable) cases versus non-COVID-19 GBS. However, the number of COVID-19 definite GBS cases ventilated was higher than all other groups $(7 / 13,54 \%$ compared to $0 / 12$ probable and $5 / 22$, $22 \%$ non-COVID-19). Despite this, the GBS disability score at four weeks was no different between all groups, suggesting the requirement for initial ventilation was secondary to active COVID-19 pulmonary involvement rather than neuromuscular weakness in PCR-positive, definite COVID-19 cases. 
There were no differences in the treatment of GBS subgroups. IVIg was the first therapy in $83 \%$ cases, and only one patient received PLEX as second line therapy. One patient received more than one course of IVIg. One patient (COVID-19 definite) died. Death was attributed to pulmonary complications rather than neuromuscular weakness.

\section{Comparison between SARS-CoV-2 and human genome and proteome}

When we examined the entire SARS CoV-2 genome [29 903 bases (b); NC_045512.2] as well as overlapping fragments of $1000 \mathrm{~b}( \pm 500 \mathrm{~b})$ and compared this to the human genome, we found no significant similarity.

We also explored individual proteins encoded by the SARS-CoV-2 genome comparing these against all referenced human proteins. Only the replicase ORF1ab/ORF1a polyprotein (7096 amino acids) produced a match with the human mono-ADP-ribosyltransferase (PARP14) protein. PARP14 belongs to an enzyme superfamily involved in histone modification during DNA damage and is ubiquitously expressed making it unlikely as a mimotope. These two proteins are $32 \%$ identical (E-value $3 \times 10^{-6}$ ) but have only one contiguous identical sequence of five or more amino acids (Val-Val-Val-Asn-Ala) that might act as a cross reactive linear peptide epitope. The remaining SARS-CoV-2 proteins including the spike/surface, envelope, membrane and nucleocapsid phosphoprotein have no significant similarity with any referenced human protein.

\section{Discussion}

Although it is profoundly difficult to prove no link in a rare disease, this retrospective epidemiological and prospective cohort study does not support any significant causal link between COVID-19 infection and GBS. ${ }^{36}$ We have used several reliable sources of data to collate the best evidence from each to demonstrate the lack of likelihood of a significant causative link. The population-based data find no plausible temporal relationship between COVID-19 and GBS (Fig. 1), a reduction in cases of GBS in comparison to preceding years (Fig. 2) and no correlation between COVID and GBS incidence at regional level (Fig. 3). There are in addition no identifiable COVID-19 associated GBS features that differentiate it from GBS in non-pandemic circumstances, in this, the largest cohort reported to date. There are also no scientific data to support a molecular mimicry link of SARS-CoV-2 to GBS at the nucleic 
acid or protein level, other than a presumptive analogy to other known bacterial and viral GBScausing pathogens. The lack of even a short, linear homology between the SARS-CoV-2 structure proteins and any axonal or myelin surface proteins reduces the likelihood that molecular mimicry with SARS-CoV-2 might be a putative mechanistic link of SARS-CoV2 to GBS.

The UK has a single highly regulated IVIg supply. IVIg is routinely available for all patients with GBS, but every vial given for GBS is logged under a mandatory NHS-based system linked directly to clinicians and to subsequent payment. The mandatory reporting to the NHSE National Immunoglobulin Database correlated with audits and clinical data showing that nearly all GBS patients receive IVIg, as well as incidence figures from this data source, which correspond exactly with reported incidence rates from multinational population-based epidemiological studies, supports the database as an appropriate repository for epidemiological analysis. Using this almost unique source we identified significantly fewer cases of GBS during the COVID-19 pandemic compared to previous years. This could represent an underascertainment of cases during lockdown for several reasons including incomplete IVIg prescription recording or patients avoiding hospital attendance.

UK Hospital Trusts are mandated to report all IVIg treatments to the database within 90 days and previous database analyses have shown $95 \%$ of cases were recorded within 30 days of treatment, and 98\% within 90 days. We collected data from 1 January to 31 May 2020 on 7 July, thus allowing sufficient time to capture the majority of reported cases. A subsequent direct check of complete IVIg reporting, specific requests for clinicians to document cases and a cross check between clinical reports and IVIg prescribing data ensured we have as complete a dataset as possible.

Mildly symptomatic patients may have decided not to visit hospital for fear of contracting COVID-19. This issue was recognized in stroke and emergency medicine with declines in overall admissions worldwide. ${ }^{37-40}$ Physicians' prescribing behaviour could also have changed during the pandemic, being more selective in patients treated, including with IVIg. However, the National Immunoglobulin Database only records GBS cases meeting criteria for treatment. The indication for IVIg treatment in GBS is for non-ambulant patients, and therefore it is unlikely such patients remained at home or would not be admitted to hospital. Even in 201619 only cases with significant disability and meeting criteria for treatment were recorded. This significantly reduces the likelihood of a disparity resulting from mild disease attendance bias, 
as a result of COVID-19 explaining the decline. Furthermore, within our cohort study $83 \%$ of cases were treated with IVIg, providing cross validation of high treatment rates, but also the fact that milder patients continued to attend to some extent.

We hypothesize that the lockdown measures introduced to prevent COVID-19 transmission have had secondary effects of reducing other common transmissible infective GBS triggers such as upper respiratory tract infections through social distancing and mask wearing, and gastrointestinal illnesses as fewer people dined out and stricter hand hygiene was adhered to. In our cohort of 47, only $1 / 47$ (2\%) reported diarrhoea preceding their GBS, significantly fewer than in the European IGOS patients at $25 \%{ }^{22}$ This is speculative but consistent with our hypothesis. Other studies have reported significant reductions in airborne or faeco-oral transmissible infectious diseases during lockdown, supporting this assertion. ${ }^{41}$ Although the true impact of hygiene measures is unknown, the avoidance of $C$. jejuni and respiratory pathogens could conceivably reduce the incidence of GBS, and may explain the pandemicrelated reduction of GBS cases. Successful interventions to lower Campylobacter contamination of fresh poultry meat have previously been reported to reduce hospitalizations for GBS by $13 \%,{ }^{42}$ and so this assertion is not impossible.

The true COVID-19 incidence in the UK is known to have been significantly under-reported. Until the end of March 2020, only patients admitted to hospital were tested for COVID-19 by PCR, and after this time it took 2 months for community testing to record symptomatic cases elsewhere. Only PCR confirmed cases were reported in the published data. Where a known link of GBS to an infectious agent exists, rates of occurrence have been published and are in the range of 0.2 to 2.2 cases per 1000 infections; for example GBS occurs at $0.25-0.65$ per 1000 cases of C. jejuni, 0.6-2.2 per 1000 cases of primary cytomegalovirus, and 0.24 per 1000 Zika virus infections. ${ }^{10,14,43}$ Utilizing PCR confirmed cases as a denominator to calculate the rate of GBS COVID occurrence suggests 0.82 cases per 1000 COVID-19 infections. As COVID-19 is a novel infection with no pre-existing seropositivity, it provides a unique opportunity to assess infection relationship rates. The true community infection rates of COVID were nearly $60 \times$ higher than the published PCR rate from the measured seroprevalence; furthermore, serological data may even still under-report the true COVID-19 infection rates as antibody responses are not detectable in all post COVID-19 infected cases, in which SARS-CoV-2 specific T-cell immune responses may occur. ${ }^{44}$ The COVID-19 seroprevalence estimated GBS incidence is 0.016 per 1000 COVID-19 infections (1.6 per 100000 , and equivalent to the usual incidence of GBS). Although it is difficult to entirely rule 
out a causative link, these data provide further evidence for a lack of strong relationship between COVID-19 and GBS compared to other recognized GBS-associated infective pathogens, and potential over-reporting of an association when using PCR confirmed cases only.

Whilst at an epidemiological level we found no increase in GBS linked to the COVID-19 epidemic, our data do not exclude the possibility that SARS-Cov-2 might be a driver of GBS in very rare cases, or that a significant reduction in non-COVID-19 GBS could mask a smaller spike of COVID GBS cases. However, other infective causes of GBS have been identified through demonstrating a peak in incidence temporally related to rises in the causative infective pathogen. With SARS-CoV-2 being one of the most prevalent infective pathogens in the last century, it is more conceivable that the absence of any increase in GBS cases during the pandemic is more likely due to a lack of causation between COVID-19 and GBS. We have also shown that there is no significant homology between any SARS-CoV-2 genetic or linear protein structure and human linear protein structures, making a molecular mimicry causation less likely. The lack of homology does not exclude immunological similarity entirely as antibody epitopes are often non-linear. Furthermore, post-translational modification of viral proteins by their host cells can occur, which theoretically could result in the generation of immunogenic surface glycomolecules so far unknown. ${ }^{45}$ Although molecular mimicry is the only fully proven pathogenic GBS mechanism, we acknowledge others could exist. More research is required to determine whether a causal relationship exists between SARS-CoV-2 and GBS.

Some small early series of COVID-19 associated GBS have been reported. ${ }^{8,46}$ The series of Gigli et $a l .{ }^{46}$ reported eight patients, all of whom were swab negative, one seropositive only and only four with COVID symptoms. Another small cohort of five reported specific disease characteristics suggesting differences from typical AIDP. ${ }^{8}$ Our prospective cohort study compared 47 cases of GBS, 13 with definite COVID-19 infection, 12 probable, and 22 with no evidence of COVID-19. Although over half of cases in our clinical cohort of GBS had evidence of COVID-19 infection, reporting bias could have influenced these proportions. A similar effect will have influenced the medical literature with over-reporting of COVID-19 GBS cases in small studies creating an impression of significant co-existence of the two conditions. The purpose of our cohort was only to compare clinical characteristics of COVID-19 and non COVID-19 associated GBS. Our cohort revealed no differences in the clinical and neurophysiological features, disease severity and outcomes of COVID-19 and non-COVID-19 
associated GBS. A larger proportion of COVID-19 PCR-positive GBS cases required mechanical intervention compared to all other groups. The similar rate of neurological recovery across all groups suggests ventilation was more related to COVID-19 associated pulmonary involvement rather than neuromuscular deficit at nadir.

This population based epidemiological study was not fully prospective but has been able to demonstrate no relationship between GBS and COVID-19 infections across the UK through the interrogation of several complementary data sources. As we explore potential COVID-19 associated neurological disease, a measured analysis of the statistical probability of rare disease occurrence in the context of a pandemic is required to investigate causation appropriately, and continue to manage non-COVID-19 neurology with the associated challenges on healthcare resources. This epidemiological and cohort study contradicts a growing number of reports postulating causation between SARS-CoV-2 and GBS, and indeed demonstrates a reduction of GBS cases. This paper alone cannot be considered definitive in ruling out SARS-CoV-2 as a cause of GBS, but further prospective data collection of COVID-19 associated GBS cases and laboratory research are required. Although prompt reporting of disease manifestations and potential associations of COVID-19 is important to inform public health decisions, robust scientific assessment to establish causality versus association is essential to evolve our understanding of this novel viral pathogen and its sequelae.

\section{Funding}

No specific funding was received toward this work. S.K. is funded by the Association of British Neurologists and Guarantors of Brain, P.M.M., M.Z., A.S.C., M.P.L. are supported by the National Institute for Health Research (NIHR) University College London Hospitals (UCLH) Biomedical Research Centre (BRC). The views expressed are those of the authors and not necessarily those of the funding bodies.

\section{Competing interests}

The authors report no competing interests. 


\section{Supplementary material}

Supplementary material is available at Brain online.

\section{References}

1. WHO. Timeline of WHO's response to COVID-19. World Health Organisation. https://www.who.int/news/item/29-06-2020-covidtimeline

2. Li YC, Bai WZ, Hashikawa T. The neuroinvasive potential of SARS-CoV2 may play a role in the respiratory failure of COVID-19 patients. $J$ Med Virol. 2020;92(6):552-555. doi:10.1002/jmv.25728

3. Mao L, Jin H, Wang M, et al. Neurologic Manifestations of Hospitalized Patients with Coronavirus Disease 2019 in Wuhan, China. JAMA Neurol. 2020;77(6):683-690. doi:10.1001/jamaneurol.2020.1127

4. Varatharaj A, Thomas N, Ellul MA, et al. Neurological and neuropsychiatric complications of COVID-19 in 153 patients: a UK-wide surveillance study. The Lancet Psychiatry. Published online 2020. doi:10.1016/S2215-0366(20)30287-X

5. Ghannam M, Alshaer Q, Al-Chalabi M, Zakarna L, Robertson J, Manousakis G. Neurological involvement of coronavirus disease 2019: a systematic review. J Neurol. Published online June 2020:1. doi:10.1007/s00415-020-09990-2

6. Paterson RW, Brown RL, Benjamin L, et al. The emerging spectrum of COVID-19 neurology: clinical, radiological and laboratory findings. Brain. Published online July 2020. doi:10.1093/brain/awaa240

7. Zhao H, Shen D, Zhou H, Liu J, Chen S. Guillain-Barré syndrome associated with SARS-CoV-2 infection: causality or coincidence? Lancet Neurol. 2020;19(5):383-384. doi:10.1016/S1474-4422(20)30109-5

8. Toscano G, Palmerini F, Ravaglia S, et al. Guillain-Barré Syndrome Associated with SARS-CoV-2. N Engl J Med. Published online April 2020. doi:10.1056/nejmc2009191

9. Lehmann HC, Hartung HP, Kieseier BC, Hughes RAC. Guillain-Barré syndrome after exposure to influenza virus. Lancet Infect Dis. 2010;10(9):643-651. 
doi:10.1016/S1473-3099(10)70140-7

10. Cao-Lormeau VM, Blake A, Mons S, et al. Guillain-Barré Syndrome outbreak associated with Zika virus infection in French Polynesia: A case-control study. Lancet. 2016;387(10027):1531-1539. doi:10.1016/S0140-6736(16)00562-6

11. Tam CC, O’Brien SJ, Petersen I, Islam A, Hayward A, Rodriguez LC. Guillain-Barré syndrome and preceding infection with Campylobacter, influenza and Epstein-Barr virus in the General Practice Research Database. PLoS One. 2007;2(4). doi:10.1371/journal.pone.0000344

12. Willison H, Yuki N. Peripheral neuropathies and anti-glycolipid antibodies. Brain. 2002;125:2591-2625.

13. Loshaj-Shala A, Regazzoni L, Daci A, et al. Guillain Barré syndrome (GBS): New insights in the molecular mimicry between C. jejuni and human peripheral nerve (HPN) proteins. J Neuroimmunol. 2015;289:168-176.

doi:10.1016/j.jneuroim.2015.11.005

14. Yuki N, Hartung H-P. Guillain-Barré Syndrome. N Engl J Med. 2012;366(24):22942304. doi:10.1056/NEJMra1114525

15. Kaida K, Ariga T, Yu RK. Antiganglioside antibodies and their pathophysiological effects on Guillain-Barré syndrome and related disorders - A review. Glycobiology. 2009;19(7):676-692. doi:10.1093/glycob/cwp027

16. Rinaldi S, Brennan KM, Kalna G, et al. Antibodies to heteromeric glycolipid complexes in Guillain-Barré syndrome. PLoS One. 2013;8(12):e82337. doi:10.1371/journal.pone.0082337

17. Devaux JJ, Odaka M, Yuki N. Nodal proteins are target antigens in Guillain-Barré syndrome. J Peripher Nerv Syst. 2012;17(1):62-71. doi:10.1111/j.15298027.2012.00372.x

18. Liu DX, Fung TS, Chong KKL, Shukla A, Hilgenfeld R. Accessory proteins of SARSCoV and other coronaviruses. Antiviral Res. 2014;109(1):97-109. doi:10.1016/j.antiviral.2014.06.013

19. European Centre for Disease Prevention and Control. COVID-19 Situation Update Worldwide, as of 29th September 2020. 2020. https://www.ecdc.europa.eu/en/covid- 
19/situation-updates

20. Foster M. Immunoglobulin Database Annual Report 2017/18. 2017.

http://igd.mdsas.com/wp-

content/uploads/ImmunoglobulinDatabaseReport201718_v1.pdf

21. Department of Health. Clinical Guidelines for Immunoglobulin Use: Update to Second Edition. 2011.

https://assets.publishing.service.gov.uk/government/uploads/system/uploads/attachmen t_data/file/216671/dh_131107.pdf

22. Doets A, Verboon C, Berg B Van Den, Brain TH-, 2018 U. Regional variation of Guillain-Barré syndrome. Brain. 2018;141(10):2866-2877.

23. Uncini A, Vallat J-M, Jacobs BC. Guillain-Barré syndrome in SARS-CoV-2 infection: an instant systematic review of the first six months of pandemic. J Neurol Neurosurg Psychiatry. 2020;0:1-6. doi:10.1136/jnnp-2020-324491

24. Connors E, Sutherland E. Coronavirus (COVID-19) Infection Survey - Office for National Statistics[Internet]. Off. Natl. Stat. 2020[cited 2020 Sep 15] Available from: https://www.ons.gov.uk/peoplepopulationandcommunity/healthandsocialcare/conditio nsanddiseases/bulletins/coronaviruscovid19infectionsurveypilot/latest

25. Public Health England. Sero-surveillance of COVID-19 [Internet]. 2020[cited 2020 Jul 13] Available from: https://www.gov.uk/government/publications/national-covid-19surveillance-reports/sero-surveillance-of-covid-19

26. European center for disease prevention and control. Case Definition for Coronavirus Disease 2019 (COVID-19), as of 29 May 2020.; 2020.

https://www.ecdc.europa.eu/en/covid-19/surveillance/case-definition

27. Ellul MA, Benjamin L, Singh B, et al. Neurological associations of COVID-19. Lancet Neurol. 2020;19(9):767-783. doi:10.1016/S1474-4422(20)30221-0

28. Breslow NE, Day NE. Statistical methods in cancer research. Volume II--The design and analysis of cohort studies. IARC Sci Publ. 1987;(82):1-406. doi:10.1136/jech.43.1.92-a

29. Office for National Statistics. Estimates of the population for the UK, England and Wales, Scotland and Northern Ireland - Office for National Statistics [Internet]. 
2019[cited 2020 Jul 16] Available from: https://www.ons.gov.uk/peoplepopulationandcommunity/populationandmigration/pop ulationestimates/datasets/populationestimatesforukenglandandwalesscotlandandnorthe rnireland

30. Sejvar JJ, Baughman AL, Wise M, Morgan OW. Population incidence of GuillainBarré syndrome: A systematic review and meta-analysis. Neuroepidemiology. 2011;36(2):123-133. doi:10.1159/000324710

31. Public Health England. Coronavirus (COVID-19) in the UK [Internet]. 2020a[cited 2020 Jul 13] Available from: https://www.gov.uk/government/publications/nationalcovid-19-surveillance-reports/sero-surveillance-of-covid-19

32. Sejvar JJ, Kohl KS, Gidudu J, et al. Guillain-Barré syndrome and Fisher syndrome: Case definitions and guidelines for collection, analysis, and presentation of immunization safety data. Vaccine. 2011;29(3):599-612. doi:10.1016/j.vaccine.2010.06.003

33. Fokke C, Van Den Berg B, Drenthen J, Walgaard C, Van Doorn PA, Jacobs BC. Diagnosis of Guillain-Barré syndrome and validation of Brighton criteria. Brain. 2014;137(1):33-43. doi:10.1093/brain/awt285

34. Williamson EJ, Walker AJ, Bhaskaran K, et al. OpenSAFELY: factors associated with COVID-19 death in 17 million patients. Nature. Published online July 2020. doi:10.1038/s41586-020-2521-4

35. Docherty AB, Harrison EM, Green CA, et al. Features of 16,749 hospitalised UK patients with COVID-19 using the ISARIC WHO Clinical Characterisation Protocol. medRxiv. 2020;10:2020.04.23.20076042. doi:10.1101/2020.04.23.20076042

36. Lucas RM, McMichael AJ. Public Health Classics Association or Causation: Evaluating Links between 'Environment and Disease'. Bull World Health Organ. 2005;83(10):792-795.

37. Aguiar de Sousa D, Sandset EC, Elkind MS V. The Curious Case of the Missing Strokes During the COVID-19 Pandemic. Stroke. 2020;51:1921-1923. doi:10.1161/strokeaha.120.030792

38. Perry R, Banaras A, Werring DJ, Simister R. What has caused the fall in stroke 
admissions during the COVID-19 pandemic? J Neurol. 2020;1:3. doi:10.1007/s00415020-10030-2

39. Markus HS, Brainin M. COVID-19 and stroke-A global World Stroke Organization perspective. Int J Stroke. 2020;15(4):361-364. doi:10.1177/1747493020923472

40. Public Health England. Emergency Department Syndromic Surveillance System Week 23[Internet]. 2020. Available from:

https://assets.publishing.service.gov.uk/government/uploads/system/uploads/attachmen t_data/file/891440/EDSSSBulletin2020wk23.pdf.

41. Angoulvant F, Ouldali N, Yang DD, et al. COVID-19 pandemic: Impact caused by school closure and national lockdown on pediatric visits and admissions for viral and non-viral infections, a time series analysis. Clin Infect Dis. Published online 2020. doi:10.1093/cid/ciaa710

42. Baker MG, Kvalsvig A, Zhang J, Lake R, Sears A, Wilson N. Declining GuillainBarré syndrome after campylobacteriosis control, New Zealand, 1988-2010. Emerg Infect Dis. 2012;18(2):226-233. doi:10.3201/eid1802.111126

43. Orlikowski D, Porcher R, Sivadon-Tardy V, et al. Guillain-barré syndrome following primary cytomegalovirus infection: A prospective cohort study. Clin Infect Dis. 2011;52(7):837-844. doi:10.1093/cid/cir074

44. Sekine T, Perez-Potti A, Rivera-Ballesteros O, et al. Robust T cell immunity in convalescent individuals with asymptomatic or mild COVID-19. bioRxiv. Published online June 2020:2020.06.29.174888. doi:10.1101/2020.06.29.174888

45. Mary B, Maurya S, Arumugam S, Kumar V, Jayandharan GR. Post-translational modifications in capsid proteins of recombinant adeno-associated virus (AAV) 1-rh10 serotypes. FEBS J. 2019;286(24):4964-4981. doi:10.1111/febs.15013

46. Gigli GL, Bax F, Marini A, et al. Guillain-Barré syndrome in the COVID-19 era: just an occasional cluster? J Neurol. Published online 2020. doi:10.1007/s00415-02009911-3 
Figure legends

Figure 1 Numbers of new daily COVID-19 infections from February to May inclusive, 2020 (red line) compared to Guillain-Barré syndrome cases in the UK between February to May inclusive from 2016 to 2020 (years depicted by colours in legend).

Figure 2 Monthly incidence of Guillain-Barré syndrome per 100000 individuals treated with IVIg in the UK between January and May inclusive for 2016-20.

Figure 3 Heat map of regional incidences of GBS and COVID-19 infections per 100000 across the UK from January to May inclusive, 2020. 


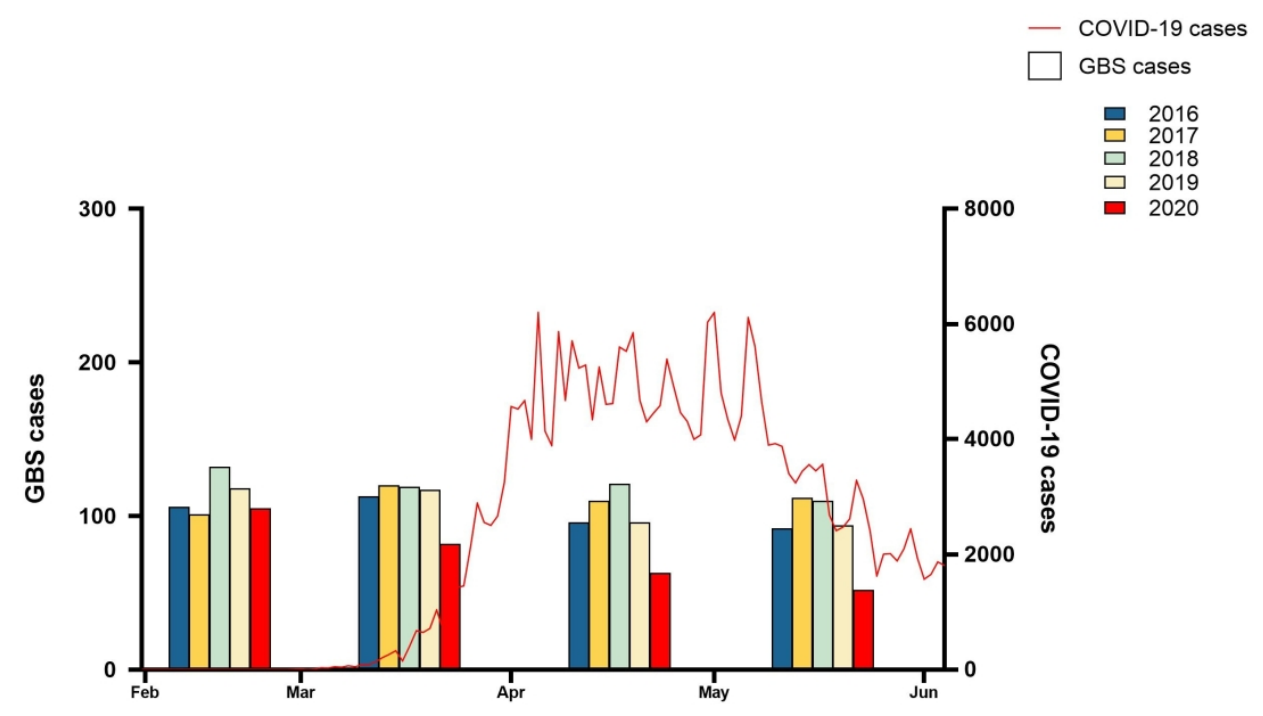

Figure 1: Numbers of new daily COVID-19 infections from February to May inclusive, 2020 (red line) compared to Guillain-Barré syndrome cases in the UK between February to May inclusive from 2016 to 2020 (years depicted by colours in figure legend).

$219 \times 124 \mathrm{~mm}(300 \times 300 \mathrm{DPI})$ 


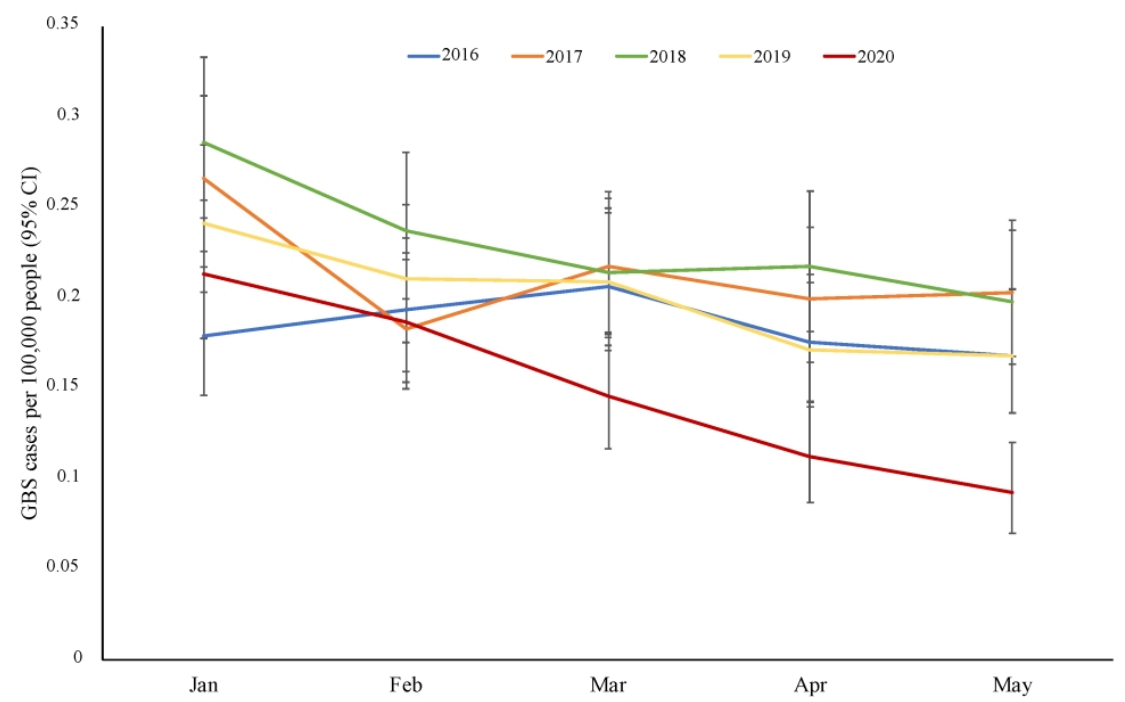

Figure 2: Monthly incidence of Guillain-Barré syndrome per 100,000 people treated with IVIg in the UK between January and May inclusive for 2016-2020.

$297 \times 209 \mathrm{~mm}(300 \times 300$ DPI $)$ 

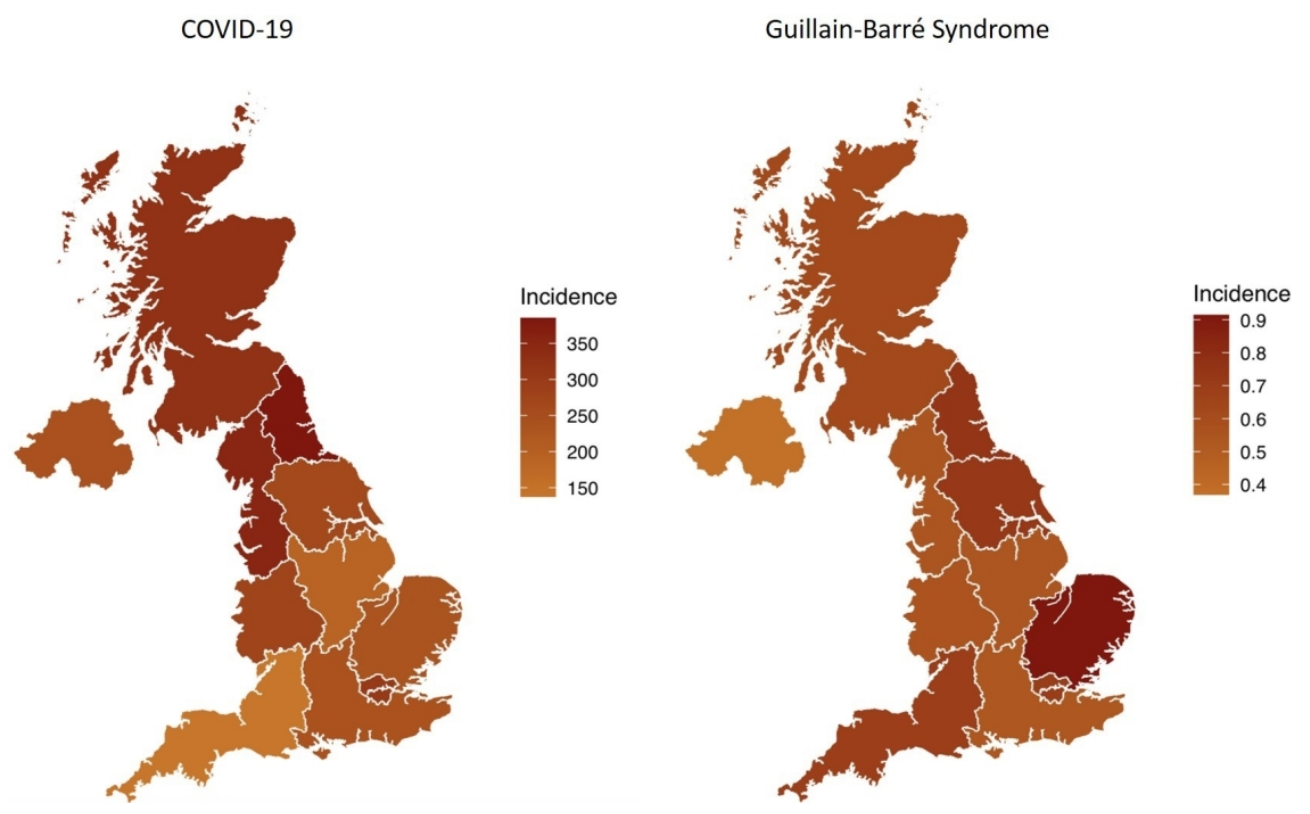

Figure 3: Heatmap of regional incidences of Guillain-Barré syndrome and COVID-19 infections per 100,000 across the UK from January to May inclusive, 2020.

$451 \times 277 \mathrm{~mm}(96 \times 96 \mathrm{DPI})$ 